RITA ADRIANA SOUZA SILVA VIEIRA

\title{
INFLUÊNCIA DE ESTÍMULOS EMOCIONAIS SOBRE A CAPTURA DA ATENÇÃO
}

Dissertação apresentada ao Programa de Pós-Graduação em Fisiologia e Biofísica do Instituto de Ciências Biomédicas da Universidade de São Paulo, para obtenção do Título de Mestre em Ciências.

Área de concentração: Neurofisiologia Orientador: Prof. Dr. Ronald D. P. K. Clive Ranvaud

SÃO PAULO

2007 


\section{RESUMO}

SOUZA-VIEIRA, R. A. Influência de Estímulos emocionais sobre a captura da atenção. 2007. 47 f. Dissertação (Mestrado em Fisiologia Humana) - Instituto de Ciências Biomédicas, Universidade de São Paulo, São Paulo, 2007.

O objetivo principal do presente estudo foi investigar se as imagens de instrumentais e da rotina Odontológica podem produzir alterações no estado atencional de voluntários, avaliado por tempos de reação escolha, como encontrado com outros estímulos emocionais, ou seja, positivos como por exemplo a visualização de alimentos, cenas de sexo; e negativos tais como imagens de sangue, cenas de violência entre outras Foram utilizados 24 sujeitos, entre 18 e 30 anos de idade, de ambos os sexos, alunos da graduação e pós graduação da Universidade de São Paulo. Propusemos estudar o impacto de imagens da rotina Odontológica com variações de um protocolo experimental já testado, em que o sujeito experimental deveria realizar um teste de discriminação entre duas barras apresentadas bilateralmente na periferia do campo visual, tendo que julgar se as barras eram ou não paralelas. Uma figura central (pertencente a uma das três categorias que foram utilizadas: Neutra, Negativa ou Odontológica) era apresentada entre as barras por um tempo curto, 200 ms. Foram ainda comparados o desempenho dos sujeitos experimentais em cada uma das categorias para avaliar o efeito da situação Odontológica comparado a situação Neutra e a Negativa. Quanto mais emocionalmente "carregada" era a figura, maior foi o tempo empregado na execução da tarefa e maior a probabilidade de erro. Na situação Negativa e Odontológica, houve um aumento no Tempo de Reação, bem como um aumento do número de erros em relação à situação Neutra. Estes resultados corroboram a proposta inicial deste projeto mostrando que as imagens Odontológicas e Negativas causam um efeito semelhante nos voluntários, ou seja, a situação Odontológica produz uma lentificação no escolha como conseqüência de uma alteração no estado atencional.

Palavras-chave: Atenção. Emoções. Estímulos emocionais. Situação Odontológica. Tempo de Reação. 


\begin{abstract}
SOUZA-VIEIRA, R. A. The influence of emotional stimulius on the capture of attention. 2007. $47 \mathrm{f}$. Master thesis (Science) - Instituto de Ciências Biomédicas, Universidade de São Paulo, São Paulo, 2007.

The principal objective of this study was to investigate, measuring choice reaction times, whether images depicting odontological routine can capture the attention of volunteers, as was demonstrated with other emotionally charged stimuli (positive: food, Sex; and negative: blood, violence). Twenty four subjects participated, both men and women, between 18 and 30 years old, all graduate or undergraduate students at the University of São Paulo. We proposed to study the impact of odontologic images with a variation of a published protocol, in which subjects were asked to decide whether two bars, one on each side of a centrally presented picture, were parallel or not. A central figure (pertaining to one of three categories, Neutral, Negative or Odontological) was presented between the two lateral bars for a short period of time, $200 \mathrm{~ms}$. The performance of the subjects was compared for each one of these categories of pictures to evaluate the effect of Odontological pictures compared with Neutral and Negativ pictures. The higher the emotional content of the picture, the slower were the responses, and the higher the incidence of errors. With both Negative and Odontological pictures responses were slower and more errors were committed than in with Neutral pictures. These results corroborate the initial proposition of this study, showing that indeed Odontological and Negative pictures had similar effects on volunteers, slowing their responses as a result of capturing their attention.
\end{abstract}

Key words: Attention. Emotions. Emotional stimulius. Odontology Situation. Reaction Time. 


\section{INTRODUÇÃO}

Há muito tempo que diferentes aspectos da atenção vem sendo objeto de estudo. Uma das definições clássicas de atenção é a proposta por William James (1890/1950) , segundo esse autor:

"Every one knows what attention is. It is the taking possession by the mind, in clear and vivid form, of one out of what seem several simultaneously possible objects or trains [p. 404] of thought. Focalization, concentration, of consciousness are of its essence. It implies withdrawal from some things in order to deal effectively with others, and is a condition which has a real opposite in the confused, dazed, scatterbrained state which in French is called distraction, and Zerstreutheit in German."

Segundo essa definição haveria um descarte de alguns estímulos para haver um processamento "preferencial" de outros; esse processo de "filtragem" dos estímulos mais relevantes para o organismo é o que tem sido denominado atenção.

A atenção permite que uma determinada informação atinja um nível elevado de processamento, ao mesmo tempo em que evita que outras informações diferentes daquela a qual se presta atenção, atinja o mesmo nível de processamento.

A maioria das teorias que tentam explicar o fenômeno da atenção visual assume que sua função seria a de facilitar seletivamente a percepção de estímulos de maneira rápida e precisa (POSNER, 1980; YANTIS, 1996), dessa maneira prestar atenção a um estímulo promove um melhor processamento do mesmo e consequentemente aumenta as chances desse estímulo ser percebido e, eventualmente, de uma resposta adequada ser executada, A atenção pode ser mobilizada de duas formas distintas:

- Atenção automática: onde a atenção do indivíduo é atraída involuntariamente para um estímulo que ocorre inesperadamente no ambiente.

- Atenção voluntária: situação na qual há um deslocamento voluntário da atenção por indicação de uma pista, por exemplo o aparecimento de uma flecha ou instruções inscritas.

Posner e Synder (1980) propõem uma teoria que distingue a atenção automática (involuntária) da atenção consciente (voluntária). Segundo eles a atenção automática 
independe do indivíduo, não é consciente, não depende de aprendizagem anterior e não seria alterada pela expectativa do sujeito ou pela memória, ocorrendo em paralelo com outros processos mentais; já a atenção voluntária é consciente e pode ser influenciada por outros processos mentais, como a expectativa do indivíduo e pela memória.

Um dos métodos clássicos de se estudar a atenção é através do método psicofísico dos tempos de reação; essa metodologia vem sendo empregada desde meados do século XIX (DONDERS, 1868), podendo tal tempo ser medido através de diferentes paradigmas experimentais (TEICHNER, 1954; LUCE, 1986; WELFORD, 1980), a saber:

- Tempo de Reação simples, medido em situação em que uma única resposta deve ser dada o mais rapidamente possível toda vez que um dado estímulo aparecer.

- $\quad$ Tempo de Reação vai / não vai, onde uma resposta deve ser dada também o mais rapidamente possível, mas apenas a alguns dentre vários estímulos que podem aparecer.

- Tempo de Reação escolha, onde o indivíduo tem que escolher uma resposta corretamente dentre várias possíveis (mais uma vez, o mais rapidamente possível), em função do estímulo que aparecer a cada tentativa.

Destes, os tempos de reação simples são os mais rápidos e os tempos de reação escolha são os que, em geral, apresentam maior latência entre estímulo e respostas.

Simons e Chabris (1999) descrevem que quanto maior a dificuldade da tarefa principal, os sujeitos engajam sua atenção na tarefa e são incapazes de relatar a ocorrência de um evento saliente, este fenômeno é conhecido como "cegueira atencional", Inattentional blindness (CHUN e MAROIS, 2002).

Uma questão em aberto é o quanto estímulos emocionais podem ser ignorados quando os sujeitos estão engajados em uma tarefa de alta demanda atencional.

Diversas evidências apontam para o favorecimento, durante o processamento sensorial, de estímulos com conteúdo emocional retiradas do IAPS (International Affective Picture System). 
O IAPS é um banco de fotografias padronizadas (LANG, 1999), divididas em três categorias :

- Figuras Neutras: Provocam um nível médio de alerta e prazer.

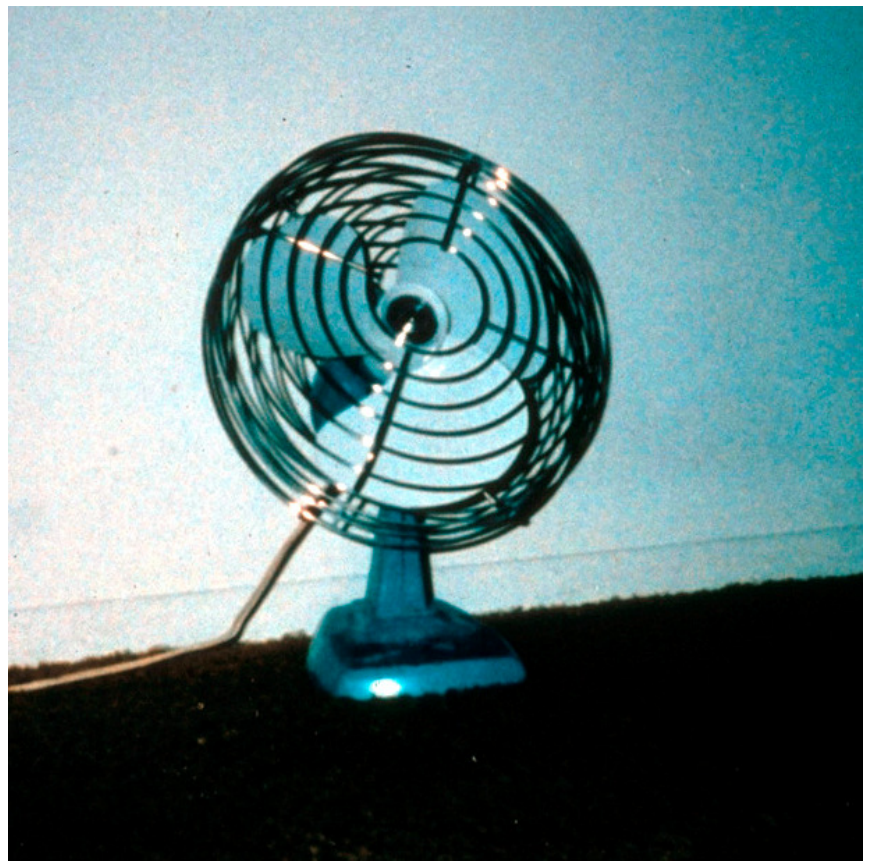

Figura 1- Figura n. 7020 do IAPS (Ventilador).

- Figuras Negativas: Provocam um alerta alto e um nível muito baixo de prazer.

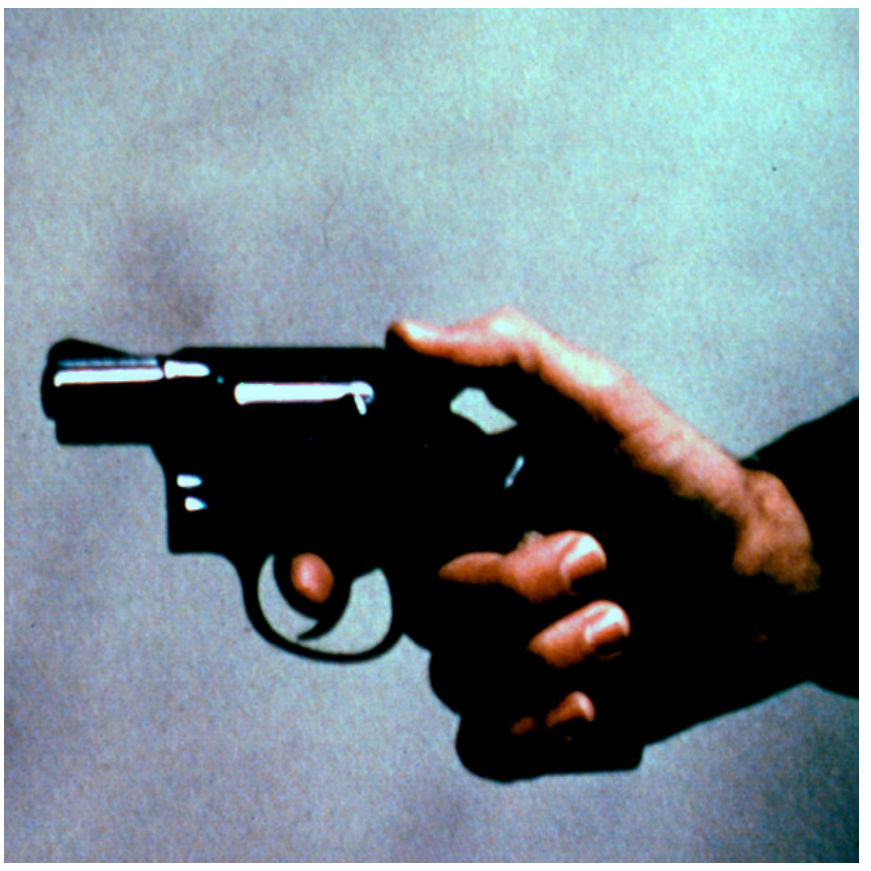

Figura 2 - Figura n. 6610 do IAPS (Arma). 
- Figuras Positivas: Provocam um nível médio de alerta e um valor alto de prazer.

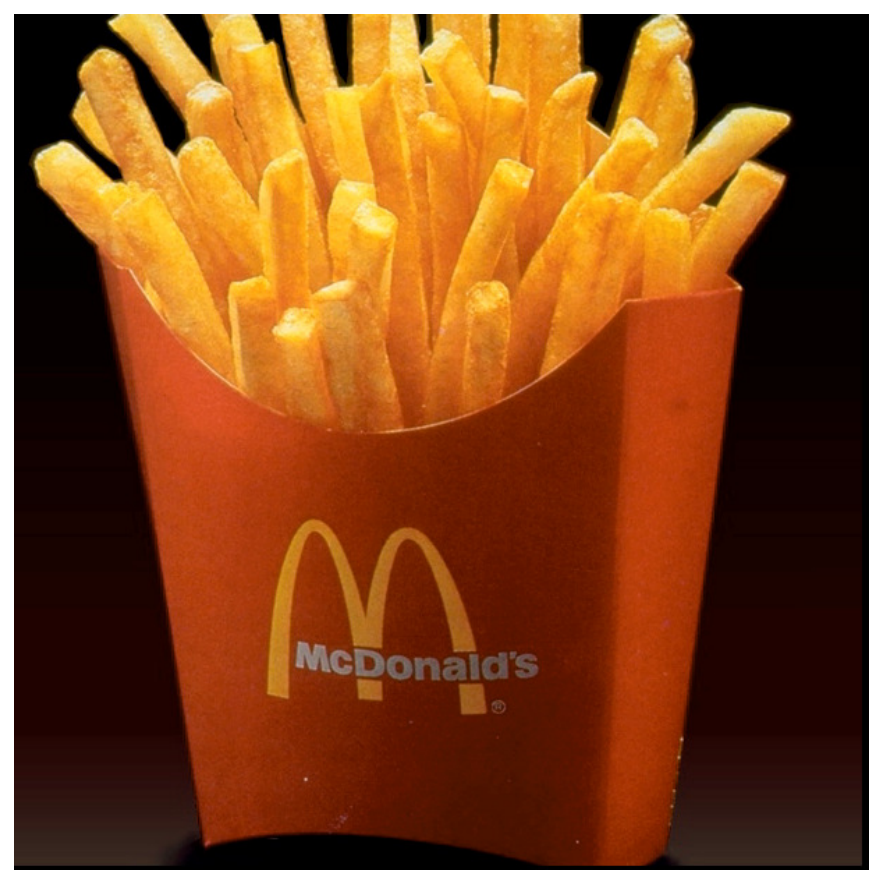

Figura 3 - Figura n. 7460 do IAPS (Batata Frita).

Esta classificação de figuras do IAPS, em Neutras, Negativas e Positivas foi obtida através de testes, onde as figuras eram apresentadas aos voluntários e estes após observá-las classificavam-nas com valores de 1 a 10 para prazer; e 1 a 10 para nível de alerta.

Volchan et al. (2003) a partir de estudos de neuroimagem encontraram maior ativação do córtex visual quando eram apresentadas figuras com conteúdo emocional do que quando eram apresentadas figuras Neutras, ou seja, os estímulos emocionais teriam vantagem no processamento neural.

Já para Schuup et al. (2003) "O processamento sensorial dos estímulos parece ser facilitado por uma atenção seletiva natural que opera em níveis precoces do processamento sensorial". Assim a emoção desempenharia um papel de fundamental importância, que seria a preparação do organismo para a aproximação (estímulos apetitivos) ou para a fuga (estímulos aversivos); e para que a resposta motora seja apropriada é necessário que o organismo detecte os estímulos relevantes.

Figuras emocionais, ou seja, figuras Negativas ou Positivas, produzem uma interferência na tarefa subsequente (BRADLEY et al., 1996). Segundo estes autores 
A visualização de imagens que tenham relação com morte, como por exemplo, cemitério, cruz, pessoas mortas, mutilação ou a visualização de sangue, (estímulos com conteúdo emocional negativo), são estímulos importantes para a evocação do medo nos seres humanos (MARKS, 1987).

Moraes e Pessoti (1988) estudaram psicologia da dor; e o efeito da ansiedade e do medo. Relatam que muitos estudiosos indicam que lidar com dor significa lidar com ansiedade e medo, necessariamente envolvidos. Afirmam também que quanto maior a ansiedade do paciente maior será sua sensibilidade à dor

Segundo o Código Internacional de Doenças, Organizacion Mundial de la Salud (1997), o medo aos cuidados odontológicos estão inseridos como fobias específicas, juntamente com ver sangue ou ferimentos, na categoria de número F40.2, Transtornos Fóbicos Ansiosos.

Segundo Cruz et al. (1997) o medo esta fortemente associado à imagem do cirurgião-dentista aparecendo de maneira mais marcante em pessoas de 20 a 30 anos, e está principalmente relacionado à visualização do instrumental. Segundo estes autores a dor também esta associada a imagem do dentista com uma elevada frequência; e indivíduos de 20 a 30 anos associam a dor principalmente ao instrumental.

Rocha et al. (2000) afirmam que ir ao dentista foi considerado o segundo entre os medos e temores mais freqüentes da população em um estudo onde os sujeitos eram questionados quanto ao seu maior medo. Quanto aos sinais e manifestações do medo, eles citam que a dilatação das pupilas, a palidez da pele, a sensação de formigamento das extremidades, choro e distúrbios gastrointestinais fazem parte do quadro sintomatológico. Em ocasiões onde o perigo não é tão evidente, mas se manifesta de forma sutil e continuada, instala-se uma apreensão no paciente, que a partir daí tornase ansioso. Como por exemplo, o paciente torna-se ansioso pelo simples fato de estar na sala de espera aguardando para ser atendido.

Canto-Pereira (1997) sugere que o foco atencional de pacientes sob atendimento odontológico seria o fator de maior importância para a modulação da resposta dolorosa frente à estimulação clínica de polpa dentária, e que as "dicas" ambientais (características do contexto odontológico) desempenhariam papel fundamental nesse processo. No seu experimento os sujeitos eram expostos a uma estimulação elétrica 
figuras emocionais, particularmente as Negativas, engajam a atenção diminuindo os recursos disponíveis para a tarefa subsequente.

O processamento emocional também pode interferir com tarefas atencionais não emocionais (HARTIKAINEN et al., 2000). Estes autores investigaram o efeito da apresentação de figuras com conteúdo emocional no desempenho de sujeitos submetidos a uma tarefa de discriminação visual. O sujeito deveria responder a um estímulo (triângulo) que acendia na tela após a apresentação de figuras Negativas, Positivas e Neutras, e deveria detectar a inclinação do triângulo, ou seja o ápice, (para cima ou para baixo) apertando uma das teclas que eram usadas de acordo com a inclinação do ápice.

Os autores encontraram uma lentificação no Tempo de Reação quando os sujeitos eram expostos a estímulos emocionais, em comparação à situação Neutra.

Tipples e Sharma ( 2000) descreveram uma lentificação nas respostas em tarefas de Tempo de Reação escolha pela apresentação de figuras Negativas, numa tarefa onde os sujeitos deveriam após o aparecimento de uma pista, discriminar entre as letras "L" ou "T" enquanto no centro da tela eram apresentadas figuras de conteúdo emocional Negativo e Neutro. Novamente a lentificação observada era maior para figuras com conteúdo Negativo.

Segundo Fortes (2003) a apresentação de figuras emocionais promoveu uma interferência na execução de tarefas, caracterizada por um aumento na latência das respostas motoras emitidas durante e/ou imediatamente após a visualização de figuras afetivas. Os voluntários deveriam responder o mais rapidamente possível a um estímulo visual que aparecia na tela após a apresentação de figuras com conteúdo emocional.

Trabalhos recentes (MOURÃO-MIRANDA, 2003; GÓMEZ, 2004) relatam diminuição da freqüência respiratória e lentificação dos tempos de reação em uma tarefa vai / não-vai de sujeitos submetidos a testes logo após apresentação de figuras com carga emocional Negativa.

Gomez; Stahelb; Danusera (2004) Encontraram uma diminuição da frequência respiratória e um aumento da condutância de pele em testes nos quais eram apresentadas aos sujeitos figuras Negativas. 
progressiva do dente incisivo central superior, e deveriam informar ao pesquisador quando começasse a sentir dor.

Foram realizadas 5 séries de estimulação elétrica para a determinação. A estimulação era feita em dois ambientes.

- Em um consultório dentário: os pacientes eram posicionados na cadeira Odontológica e o pesquisador paramentado fazia a estimulação do dente até que o sujeito relatasse dor (Situação Odontológica).

- E uma situação Neutra, em que o pesquisador fazia a estimulação do dente até que o sujeito relatasse dor, porém em uma sala na qual não havia materiais ou instrumentais odontológicos e o pesquisador não estava paramentado (Situação Neutra).

Este estudo possibilitou verificar a influência do ambiente sobre o limiar de percepção de dor e limiar de tolerância à dor.

A situação que simulava a de atendimento odontológico provocava maior sensibilidade dolorosa e menor tolerância à dor nos sujeitos .

Houve também maior sensibilidade dolorosa frente à primeira série de estimulação sugerindo a participação do foco de atenção do sujeito frente à estimulação dolorosa, talvez como o principal fator na modulação da resposta dolorosa.

Johnsen et al. (2003) foram além, e em um estudo com pacientes odontológicos mediram os tempos de reação em uma tarefa de leitura de palavras Neutras e palavras com conotação odontológica . Na situação onde as palavras mostradas tinham conotação odontológica houve uma lentificação no Tempo de Reação em relação à situação Neutra. 


\section{CONCLUSÃO}

Através do nosso estudo podemos concluir que:

1. O protocolo experimental se demonstrou aplicável ao estudo de emoções desencadeadas por imagens do contexto odontológico.

2. Deveria ser possível aplicar o mesmo protocolo para avaliar emoções (tanto positivas como negativas) desencadeadas por outras situações, tanto no contexto médico (situações clínicas, estudo de fobias etc.), como em outras situações da vida de todos os dias.

3. As figuras Odontológicas tiveram um impacto semelhante ao de figuras Negativas do IAPS, e maior do que figuras Neutras, claramente indicando objetivamente que o contexto odontológico mobiliza emoções poderosas, que podem em princípio ser quantificadas, o que deverá permitir a elaboração de estratégias de atendimento que minimizem o desconforto dos pacientes.

4. Atualmente a avaliação de nível de ansiedade odontológica de pacientes é avaliada apenas por questionários, fornecendo dados de certa forma subjetivos; a aplicação do presente protocolo deve viabilizar luma medida mais objetiva. 


\section{REFERÊNCIAS BIBLIOGRÁFICAS}

AGRESTI, A. Categorical data analysis. 2. ed. New York: Wiley-Interscience, 2002. $710 \mathrm{f}$.

BRADLEY, M.M.; CUTHBERT, B.N.; LANG, P.J. Picture media and emotion: effects of a sustained affective context. Psychophysiology, v. 33, n. 6, p. 662-670, 1996.

CANTO-PEREIRA, L.H.M. Influência do ambiente sobre os limiares de percepção e de tolerância à dor dentária: um estudo psicofísico. $76 \mathrm{f}$. Dissertação (Mestrado em Neurociências) - Instituto de Psicologia, Universidade de São Paulo, São Paulo, 1997.

CHUN, M.M.; MAROIS, R. The dark side of visual attention. Current Opinion in Neurobiology, v. 12, p.184-189, 2002.

CRUZ, J. S. et al. A imagem do cirurgião-dentista: um estudo de representação social. Rev. Odontol. Univ. São Paulo, v. 11, n. 4, p. 307-313, 1997.

DONDERS, F.C. On the speed of mental processes. In: KOSTER, W.G.(Ed.). Attention and performance II. Acta Psychologica, v. 30, p. 412-431, 1969.

ERTHAL, F.; VOLCHAN, E. Captura da atenção por estímulos emocionais. Paidéia, v. 14, n. 27, p. 35-44, 2003.

FORTES, M.G.P. Modulação do desempenho em uma tarefa de Tempo de Reação simples por figuras com conteúdo emocional. 193 f. Dissertação (Doutorado) Instituto de Biofísica Carlos Chagas Filho, Universidade Federal do Rio de Janeiro, 2003.

GOMEZ, P.; STAHEL, W.A.; DANUSER, B. Respiratory responses during affective picture viewing. Biological Psychology, v. 67, n. 3, p. 359-373, 2004.

HARTIKAINEN, K.M.; OGAWA, K.H.; KNIGHT, R.T. Transient interference of right hemispheric function due to automatic emotional processing. Neuropsychologia, v. 38, n. 12 , p. 1576-80, 2000.

JAMES, W. Principles of Psychology. New York: Dover, 1950. Original publicado em 1890.

JOHNSEN, B. H.; THAYER, J. F.; LABERG, J. C. Attentional and physiological characteristics of patients with dental anxiety. Journal of Anxiety Disorders, v. 17, n. 1, p. 75-87, 2003. 
LANG, P.J.; BRADLEY, M.M.; CUTHBERT, B.N. Motivated attention: Affect, activation, and action. In: SIMONS, R.F.; BALABAN, M.T.; LANG, P.J. (Ed). Attention and Orienting: Sensory and Motivational Processes, p. 97-135, 1997.

LANG, P.J.; BRADLEY, M.M.; CUTHBERT, B.N. International Affective Picture System (IAPS): Instruction Manual and Affective Ratings. Gainesville: University of Florida - The Center for Research in Psychophysiology, 1999.

LUCE, R.D. Response times. New York: Oxford University Press, 1986.

MARKS, I.M. Fear, phobias and rituals. New York: Oxford University Press, 1987.

MILGROM, P.; WEINSTEIN, P. Dental Fears in General Practice: new Guidelines for Assessment and Treatment. International Dental Journal, v. 43, n. 3, p. 288-293, 1993.

MORAES, B.A.; PESSOTI, I. A. Psicologia e a Dor. In: PAIVA, J.G.; ANTONIAZZI, J. H. (Ed.). Endodontia: Bases para a Prática Clínica. São Paulo: Artes Médicas, p. 289-297, 1988.

MOURÃO-MIRANDA, J.; VOLCHAN, E.; MOLL, J.; OLIVEIRA-SOUZA, R.; OLIVEIRA, L.; BRAMATI, I.; GATTASS, R.; PESSOA, L. Contributions of stimulus valence and arousal to visual activation while viewing emotion-laden stimuli. Neuroimage, v. 20, n. 4, p. 1955-1963, 2003.

OLDFIELD, R.C. The assessment and analysis of handedness: The Edinburgh Inventory. Neuropsychologia, v. 9, p. 97-113, 1971.

ORGANIZACION MUNDIAL DE LA SALUD. Organización Panamericana de la Salud. Transtornos Mentales y del Comportamiento. Clasificación Estadística Internacional de Enfermedades y Problemas Relacionados com Salud. 10. ed. Washington, DC, 1995. p. 295-325.

PAUlinO, C. D.; SINGER, J. M. Análise de dados categorizados. São Paulo: Ed. Edgard Blucher, 2006. 648f.

POSNER, M.I. Orienting of attention. Quarterly Journal of Experimental Psychology, v. 32, p.03-25, 1980.

POSNER, M.; SYNDER, C.; DAVIDSON, B. Attention and detection of signals. Journal of Experimental Psychology: General, v. 109, p. 160-174, 1980.

RIBEIRO, L.R.; POMPÉIA, S.; AMADEO-BUENO, O. F. Brasilian Norms for the International Affective Picture System (IAPS): brief report. Revista de Psiquiatria do Rio Grande do Sul, v. 26, n. 2, 2004. 
ROCHA, R.G. et al. O Medo e a Ansiedade no Tratamento Odontológico: Controle Através da Terapêutica Medicamentosa. In: FELLER, C.; GORAB, R. (Ed.). Atualização na Clínica Odontológica. São Paulo: Artes Médicas, p. 391-410, 2000.

SCHNEIDER, W.; ESCHMAN, A.; ZUCCOLOTTO, A. E-Prime User's Guide. Pittsburgh: Psychology Software Tools, (2002).

SEGALOWITZ, S.J.; GRAVES, R. Suitability of the IBM XT, AT and S/2 keyboard, mouse, and game port as response devices in reaction time paradigms. Behavior Research Methods, Instruments, and Computers, v. 22, p. 283-289, 1990.

SIMONS, D.J.; CHABRIS, C.F. Gorillas in our midst: sustained inattentional blindness for dynamic events. Perception, v. 28, p. 1059-1074, 1999.

TEICHNER, W.H.; Recent studies of simple reaction time. Psychology Bull, v. 51, p. 128-149, 1954.

TIPPLES, J.; SHARMA, D. Orienting to exougenous cues and attentional bias to affective pictures reflect separate processes. Journal Psychology., v. 91, n. 1, p. 8797, 2000.

VOLCHAN, E.; PEREIRA, M.G.; OLIVEIRA, L.D.; VARGAS, C.; MOURAOMIRANDA, J.; AZEVEDO, T.M; Machado-Pinheiro, W. Emotional stimuli: sensory processing and motorresponses. Revista Bras Psiquiatria, v. 25, p. 29-32, 2003. Suppl. 2.

WATSON, D.; CLARK, L.A.; TELLEGEN, A. Development and validation of brief measures of positive and negative affect: The PANAS Scales. Journal of Personality and Social Psychology, v. 54, n. 6, p. 1063-1070, 1988.

WELFORD, A.T. Reaction Times. London: Academic Press, 1980.

YANTIS, S. Attentional capture in vision. In: KRAMER, A.F.; COLES, G.H. (Ed.). Converging operations in the study of visual selective attention, 1996. 